\title{
Partner Character Attracting Consumers to a Real Store
}

\author{
Motoi Okuzono ${ }^{1 *}$, Masahumi Muta ${ }^{2}$, \\ Soh Masuko ${ }^{2}$, Hayaki Kawata ${ }^{1}$ and Junichi Hoshino ${ }^{1}$ \\ ${ }^{1}$ University of Tsukuba, Graduate school of Systems and Information Engineering, \\ 1-1-1, Tennodai, Tsukuba-shi, Ibaraki, Japan \\ \{motoi,kawata.hayaki\}@entcomp.esys.tsukuba.ac.jp,jhoshino@esys.tsukuba.ac.jp \\ ${ }^{2}$ Rakuten, Inc., Rakuten Institute of Technology, \\ Rakuten Crimson House, 1-14-1, Tamagawa, Setagaya-ku, Tokyo, Japan \\ \{masafumi.muta, so.masuko\}@rakuten.com
}

\begin{abstract}
The diversification of personal tastes and the rapid increase in choices makes it difficult to select things that suits them. For service providers it is fixedly segmented like a conventional mass media It is becoming more difficult to provide advertisements and services to individuals. Because of these circumstances, it is requested that we can accurately acquire personal preferences and analyze them. On the other hand, attention is focused on $\mathrm{O} 2 \mathrm{O}$, which connects online actions to offline online shopping in real stores.We also use gaming which makes various elements of everyday life into a game, Measures have also been taken to immerse and positively tackle purchasing behavior. In the marketing field, it is said that narrative is important for inducing user's behavior. Therefore, in this research, we construct a partner character system based on scenario game and verify whether we can increase purchasing behavior in a real shop by making users engage in the system using scenarios and characters that can be friends with users.
\end{abstract}

Keywords: Pertner character, O2O, Gamification

\section{Introduction}

Interests in $\mathrm{O} 2 \mathrm{O}$ are increasing. So far, they are used for promoting visits and purchasing activities at physical retail stores with email marketing and online campaigns; there are also initiatives to lead online members and internet visitors to stores and actual off-line purchasing behaviors. There are also measures being carried out to utilize gamification which turns various factors in everyday life into a form of game to draw in users and get them to proactively undertake purchasing behaviors. Moreover, in the field of marketing, having narrativity is considered important for inducing user actions [1]. Therefore in this study, a partner character system is constructed using a scenario based game, to verify whether purchasing behaviors at physical retail stores can be increased by heightening engagement of users with the system, utilizing a scenario and a character that can befriend users. In this system, preferences of users are checked using conversation based on scenarios, and scenarios 
are changed depending on actions or location information of users. For this study, bakery was chosen as target purchasing category.

\section{System Overview}

This system uses a character for its interface.

- This character uses expressions of emotional feelings (delight, anger, sorrow and pleasure) as shown on Fig. 1

- Backgrounds change depending on the scenario scene

- Texts are displayed at lower part of the screen, to make it feel as if the character is talking to users

- Users have pseudo-conversations with the character by selecting choices that are presented

The responsibilities of this front end character are;

- Controlling the story

- Estimation of user model from interactions with users

By using this front end character, the system renders a story and carries out dialogues with users. Three levels are set for the front end character depending on how engrossed a user becomes, and new stories develop as levels go up. Moreover, a character that resides on smartphones of users is used to accumulate knowledge about users, by continuously having dialogues with users. Users would convey their preferences through dialogues with the partner, as well as evaluating proposals from the character.
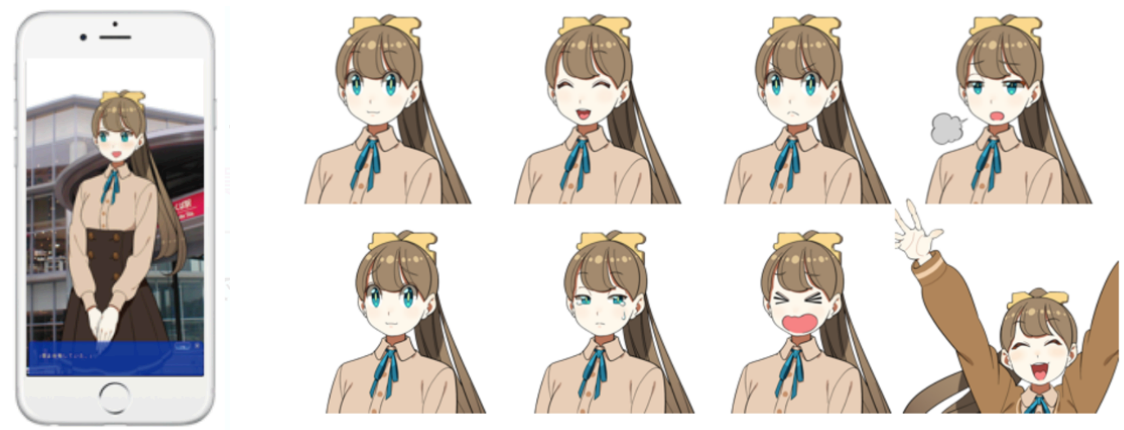

Fig. 1. Example of character expression

This system tries to lead to purchases at physical retail stores, by proposing buns that users would like. To that end, a questionnaire allowing multiple answers is carried out to 32 men and women, where favorite buns are selected out of 73 types of buns quoted from a corporate website [2] of a company that holds cooking classes on baking buns; and the results from this questionnaire are used to enable 
recommendation with a collaboration filter. When using this system for the first time, questions in Table 1 are asked by the front end character. The results from this and right or wrong of answers on quiz regarding buns that the front end character posed to users are utilized to specify buns that users would like, and based on data obtained from the aforementioned preliminary survey, collaboration filtering is carried out.

Table. 1. Initial questionnaire

\begin{tabular}{|l|}
\hline \multicolumn{1}{|c|}{ Questionnaire } \\
\hline \hline Do you often eat breads? \\
\hline Which do you like pastries or deli breads? \\
\hline Do you like sweet breads? \\
\hline Do you like breads for staple food? \\
\hline
\end{tabular}

As an engagement parameter of users to the system, a degree of closeness is implemented in this system. There are four levels in the degree of closeness including the initial stage, with several criteria for going up in the level. Criteria are;

- Visit a bakery

- Listen to trivia on buns

- Provide correct answer on quiz relating to buns

- Experience bakeries or buns that the system recommended

- Give a high evaluation to the bakery or buns that the user experienced

By going up in the level, events would proceed and the character will become friendlier in its manner of speaking to users, showing more expressions.

\section{$3 \quad$ Evaluation Testing}

Test subjects are 16 male students between the ages of 20 to 25 who are studying at University of Tsukuba. They are asked to spend a week using the system. Furthermore, while there are no rules set forth to enforce use of the system, allowing the system to use location information and to use push notification at 12:00 every day were mandatory.

Test subjects were classified based on their degree of closeness with the character, and their average number of visits to physical retail stores was summarized in Table 2. The results showed that higher the degree of closeness, the more they tended to visit stores. Therefore this showed that the more users become closer with a character in a system with narrativity, the more they visit physical retail stores, which means leading to purchasing behaviors. 

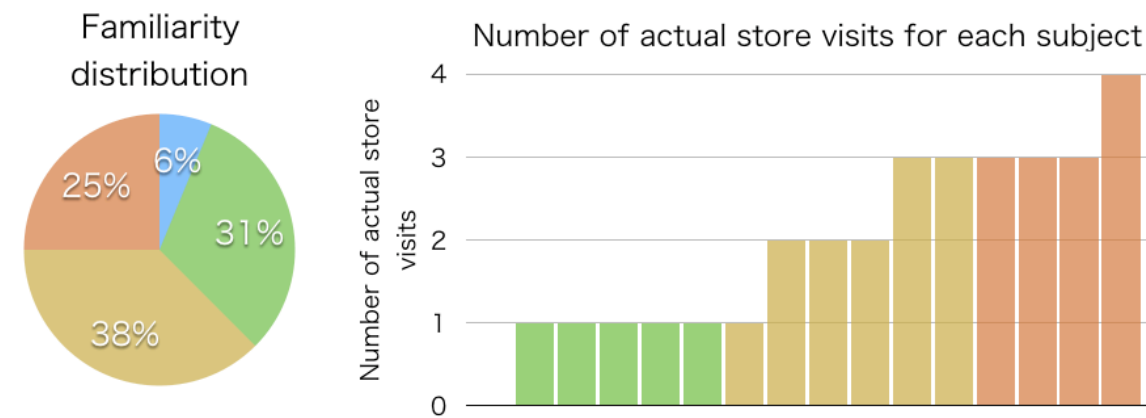

Familiarity $0 \bigcirc$ Familiarity $1 \bigcirc$ Familiarity $2 \bigcirc$ Familiarity 3

Fig. 2. Familiarity distribution and number of actual store visits for each subject

\section{Conclusion}

While it showed that higher degree of closeness resulted in attracting more customers to physical retail stores, a third of test subjects were at the initial state of closeness; therefore more contrivance is needed to heighten engagement with the system. Moreover, since the scope was limited to buns as subjects in this study, algorithm for the system to recommend buns to users had remained a simple collaborative filtering as well. In future, it would be good to collaborate with commercial facilities and such to create partner characters that span across several fields such as apparels, books and films. And as volume of contents being handled increases, creation of contents and store database that are currently being prepared manually will likely need to be automated, by collaborating with EC (electronic commerce) websites.

\section{References}

1. Shankar, Avi, Richard Elliott, Christina Goulding. "Understanding consumption: Contributions from a narrative perspective." Journal of marketing Management 17.3-4 (2001): 429-453.

2. "Book of kind of breads I COOKTWON", https://papatto-cooktown.jp/contents/kind_of_bread, 2017-04-10 